\title{
P06-03. The Buea University develops HIV clinical trial capacity within the Cental African Network on TB, HIVIAIDS and malaria, CANTAM
}

\author{
PM Ndumbe*1, E Mavoungou ${ }^{2}$, F Ntoumi $^{3}$ and OO Oukem ${ }^{4}$
}

Address: ${ }^{1}$ Faculty of Medicine, Univeristy of Buea, Buea, Cameroon, ${ }^{2}$ Medical Research Unit of Albert Schweitzer, Lamberene, Gabon, ${ }^{3}$ OCEAC, Yaounde, Cameroon and ${ }^{4}$ Centre International de Reference Chantal Biya, Yaounde, Cameroon

* Corresponding author

from AIDS Vaccine 2009

Paris, France. 19-22 October 2009

Published: 22 October 2009

Retrovirology 2009, 6(Suppl 3):P92 doi:10.1186/1742-4690-6-S3-P92

This abstract is available from: http://www.retrovirology.com/content/6/S3/P92

(c) 2009 Ndumbe et al; licensee BioMed Central Ltd.

\section{Background}

The EDCTP has currently funded the Central African Network on Tuberculosis, HIV/AIDS and Malaria for the conduct of clinical trials because of the paucity of capacity to conduct clinical trials in this region. However, the complexity of the HIV epidemic lends itself to the development of innovative technology to accelerate the utilization of new tools, such as vaccines, for its control. This is more so as this region is known to abound in genetic diversity of the HIV virus.

\section{Methods}

Within the programme, relatively strong teams are linked with weaker units to create interest groups in HIV vaccine trials and networks. This would require training of individual scientists in GCLP, the recruitment and retention of volunteers, community engagement, data management and ownership of data, and ethical considerations for the conduct of trials. It would also require structural and infrastructural development, procedural arrangemets in both the strong and weak sites. Training will be conducted both within the region and outside the region in established international clinical trial sites. For ease of retention of trainees, individuals with advanced qualifications or those with fixed positions will have an edge in the selection process so as to ensure sustainability.

\section{Results}

It is expected that within three years, individuals would have been trained in all relevant aspects of clinical trials, structural and infrastructural arrangements would have been made in the weak institutions, procedural matters would have been finalised both within and among the institutions in the network, the network would be in a position to conduct clinical trials according to international norms.

\section{Conclusion}

The diversity in HIV clades in the Central African Region makes it ideal for the conduct of quality studies on pathogenesis and vaccine trials. The EDCTP model of selectively identifying weak instutions for capacity development bears promise for retaining staff and sustaining gains. 